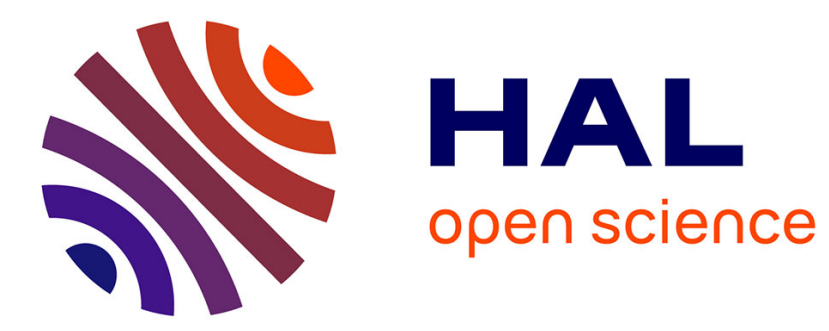

\title{
Formulation of rigid body systems with nonsmooth and multivalued interactions
}

Christoph Glocker

\section{To cite this version:}

Christoph Glocker. Formulation of rigid body systems with nonsmooth and multivalued interactions. Second World Congress on Nonlinear Analysis, 1997, Athènes, Greece. pp.4887-4892, 10.1016/S0362546X(96)00109-5 . hal-01379710

\section{HAL Id: hal-01379710 https://hal.science/hal-01379710}

Submitted on 12 Oct 2016

HAL is a multi-disciplinary open access archive for the deposit and dissemination of scientific research documents, whether they are published or not. The documents may come from teaching and research institutions in France or abroad, or from public or private research centers.
L'archive ouverte pluridisciplinaire HAL, est destinée au dépôt et à la diffusion de documents scientifiques de niveau recherche, publiés ou non, émanant des établissements d'enseignement et de recherche français ou étrangers, des laboratoires publics ou privés. 


\title{
FORMULATION OF RIGID BODY SYSTEMS WITH NONSMOOTH AND MULTIVALUED INTERACTIONS
}

\author{
CHRISTOPH GLOCKER $\ddagger$ \\ Institute of Steel Structures, Aristotle University of Thessaloniki, \\ GR-54006 Thessaloniki, Greece
}

Key words and phrases: Principles of d'Alembert, Jourdain and Gauss, Principle of Least Constraints, variational inequality, differential inclusion, convex potential function, multivalued interaction law.

\section{INTRODUCTION}

This paper treats the formulation of interaction laws in rigid multibody systems. The interaction laws may be represented by a certain class of convex $C^{0}$ potential functions and then derived through subdifferentiation. The resulting multifunctions contain the cases of smooth force characteristics, bilateral constraints, as well as combinations of them like unilateral constraints, dry friction, or prestressed springs or damper combinations. Impacts are excluded. A generalization of the principles of d'Alembert, Jourdain, and Gauss in terms of variational inequalities will be given. The paper is organized as follows. In Section 2 some preliminaries about directional derivatives and subdifferentials are stated, cp. [1]. Section 3 contains the formulation of the differential inclusions of rigid multibody systems as well as the Principle of d'Alembert. From that, and using the results of Section 2, the Principles of Jourdain and Gauss are derived in Sections 4 and 5. Finally, Section 6 contains the Principle of Least Constraints, i.e. a minimization problem in terms of the unknown accelerations.

\section{PRELIMINARIES}

A function $f: \mathbb{R}^{n} \rightarrow \mathbb{R}$ is said to be one-sided directional Gâteaux-differentiable at $x$ if there exists a $f^{\prime}(x, y)$ such that

$$
\lim _{\lambda \downarrow 0} \frac{1}{\lambda}[f(x+\lambda y)-f(x)]=f^{\prime}(x, y), \quad \forall y \in \mathbb{R}^{n} .
$$

Note that $f^{\prime}(x, y)$, if exists, is a convex, positively homogeneous function of $y$. If $f$ is convex and bounded on a neighborhood of $x$, one defines the subdifferential of $f$ at $x$ by

$$
\partial f(x)=\left\{x^{*} \mid f^{\prime}(x, y) \geq\left\langle x^{*}, y\right\rangle, \quad \forall y\right\} .
$$

With (2.2) the directional derivative (2.1) can be expressed as

$$
f^{\prime}(x, y)=\sup _{x^{*}}\left\{\left\langle x^{*}, y\right\rangle \mid x^{*} \in \partial f(x)\right\} .
$$

$\ddagger$ On leave from the Lehrstuhl B für Mechanik, TU München with a Feodor Lynen research fellowship 
Furthermore, we see from (2.2) that $x^{*} \in \partial f(x)$ if and only if the variational inequality

$$
f^{\prime}(x, y) \geq\left\langle x^{*}, y\right\rangle, \quad \forall y
$$

holds. Next we introduce the indicator function $I_{C}$ and the support function $I_{C}^{*}$ of a convex set $C$,

$$
I_{C}\left(x^{*}\right)=\left\{\begin{array}{ll}
0 & \text { for } x^{*} \in C \\
+\infty & \text { for } x^{*} \notin C
\end{array} \quad I_{C}^{*}(y)=\sup _{x^{*}}\left\{\left\langle y, x^{*}\right\rangle \mid x^{*} \in C\right\}\right.
$$

which are connected together through conjugacy. Hence, we can write Fenchel's inequality in the form

$$
\left\langle y, x^{*}\right\rangle \leq I_{C}^{*}(y), \quad \forall y, \quad \forall x^{*} \in C,
$$

which holds as an equality if and only if $y$ is a subgradient of $I$ at $x^{*}$ or vice versa,

$$
\left\langle y, x^{*}\right\rangle=I_{C}^{*}(y) \Leftrightarrow y \in \partial I_{C}\left(x^{*}\right) \Leftrightarrow x^{*} \in \partial I_{C}^{*}(y) .
$$

Now we will determine the set of $\bar{x}^{*}$ for which eq. (2.4) holds as an equality if $y$ is fixed. For that we consider the directional derivative $f^{\prime}(x, y)$ from (2.3) as a function of $y$,

$$
g(y)=f^{\prime}(x, y)=\sup _{x^{*}}\left\{\left\langle x^{*}, y\right\rangle \mid x^{*} \in \partial f(x)\right\},
$$

and, comparing (2.8) and (2.5), we observe that it is the support function of the subdifferential $\partial f(x)$. Hence, the elements $\bar{x}^{*}$ for which eq. (2.4) holds as an equality are determined by (2.7),

$$
g(y)=\left\langle\bar{x}^{*}, y\right\rangle \Leftrightarrow \bar{x}^{*} \in \partial g(y) .
$$

Furthermore, we see from (2.8) and (2.9) that the set $\partial g(y)$ can be stated as

$$
\partial g(y)=\left\{\bar{x}^{*} \in \partial f(x) \mid\left\langle\bar{x}^{*}, y\right\rangle=\sup \left\langle x^{*}, y\right\rangle, \quad x^{*} \in \partial f(x)\right\} .
$$

Note that $\partial g(y) \subseteq \partial f(x)$ and $\partial g(0)=\partial f(x)$. By eq. (2.10) we have shown that the subdifferential of the directional derivative $f^{\prime}(x, y)$ as a function of $y$ consists of those elements of the subdifferential of $f(x)$ for which the equality $f^{\prime}(x, y)=\left\langle\bar{x}^{*}, y\right\rangle$ holds [1]. This result will be used in Sections 4 and 5 for calculating the velocities and accelerations potential functions from a displacement potential.

\section{THE PRINCIPLE OF D'ALEMBERT}

We consider a dynamical system consisting of a certain number of rigid bodies. The generalized displacements of the system may be described by a coordinate vector $\boldsymbol{q} \in \mathbb{R}^{f}$ which depends on time $t$. The trajectories $\boldsymbol{q}: \mathbb{R} \rightarrow \mathbb{R}^{f}$ are assumed to be absolutely continuous such that the one-sided first and second time derivatives $\dot{\boldsymbol{q}}^{ \pm}, \ddot{\boldsymbol{q}}^{ \pm}$exist at every $t$. After stating the equilibrium of the linear and angular momenta and forces the system is supposed to have the form (see [2])

$$
\boldsymbol{M}(\boldsymbol{q}) \ddot{\boldsymbol{q}}^{+}-\boldsymbol{h}\left(\boldsymbol{q}, \dot{\boldsymbol{q}}^{+}\right)=\boldsymbol{f}^{+}
$$

with a symmetric positive definite mass matrix $\boldsymbol{M}$ and a force vector $\boldsymbol{h}$ which is assumed to be a continuous function of $(\boldsymbol{q}, \dot{\boldsymbol{q}})$. This vector consists of the gyroscopical accelerations and 
may contain additional applied forces. The right hand member of (3.1) may be derived from a convex lower semi continuous potential function $V(\boldsymbol{q})$ by subdifferentiation [3],

$$
-\boldsymbol{f}^{+} \in \partial V(\boldsymbol{q})
$$

where $\partial V(\boldsymbol{q})$ denotes the subdifferential of $V$ at $\boldsymbol{q}$, see (2.2). Throughout the paper we will restrict ourself to polyhedral convex potential functions $V(\boldsymbol{q})$ only. These are convex functions whose epigraphs are polyhedral convex sets, i.e. convex sets obtained by the intersection of a finite number of closed half spaces [1]. Note that "vertical" half spaces are also allowed which correspond to affine unilateral and bilateral constraints. Combining (3.1) and (3.2) we get the differential inclusions of the system [4],

$$
-\left(\boldsymbol{M} \ddot{\boldsymbol{q}}^{+}-\boldsymbol{h}\right) \in \partial V(\boldsymbol{q}),
$$

which need some additional remarks. Clearly, the superscripts " +" can be replaced by " -" in order to take into account the velocities and accelerations from the past. Impacts are generally excluded by the formulation (3.3) because it is based on the equilibrium of momenta and forces. If impacts occure one must replace eq. (3.3) by the corresponding measure differential inclusion (see [5], [6]) because accelerations do not exist at the impact itself. However, the formulation (3.3) contains the taking off of the system from a "closed" unilateral constraint as well as the bilaterally constrained impact free motion. If the epigraph of $V$ contains no "vertical" half spaces, then the forces $\boldsymbol{f}$ from (3.2) are bounded and hence the motion is impact free, $\dot{\boldsymbol{q}}^{+}=\dot{\boldsymbol{q}}^{-}$. If, in addition, $V(\boldsymbol{q})$ is differentiable for all $\boldsymbol{q}$, then $\partial V(\boldsymbol{q})=\nabla V(\boldsymbol{q})$, in our case being the gradient of a hyperplane, and hence $\ddot{\boldsymbol{q}}^{+}=\ddot{\boldsymbol{q}}^{-}$.

With the help of (2.4) the inclusion (3.2) can also be expressed by means of a variational inequality

$$
-\boldsymbol{f}^{+} \in \partial V(\boldsymbol{q}) \Leftrightarrow V^{\prime}\left(\boldsymbol{q}, \boldsymbol{q}^{*}-\boldsymbol{q}\right) \geq-\boldsymbol{f}^{+T}\left(\boldsymbol{q}^{*}-\boldsymbol{q}\right), \quad \forall \boldsymbol{q}^{*}
$$

which leads together with (3.1) to the Principle of d'Alembert [4],

$$
-\left(\boldsymbol{q}^{*}-\boldsymbol{q}\right)^{T}\left(\boldsymbol{M} \ddot{\boldsymbol{q}}^{+}-\boldsymbol{h}\right) \leq V^{\prime}\left(\boldsymbol{q}, \boldsymbol{q}^{*}-\boldsymbol{q}\right), \quad \forall \boldsymbol{q}^{*} .
$$

Due to the convexity of $V$ the additional property $V^{\prime}\left(\boldsymbol{q}, \boldsymbol{q}^{*}-\boldsymbol{q}\right) \leq V\left(\boldsymbol{q}^{*}\right)-V(\boldsymbol{q})$ enables us to state this principle in terms of the potential function itself,

$$
-\left(\boldsymbol{q}^{*}-\boldsymbol{q}\right)^{T}\left(\boldsymbol{M} \ddot{\boldsymbol{q}}^{+}-\boldsymbol{h}\right) \leq V^{\prime}\left(\boldsymbol{q}, \boldsymbol{q}^{*}-\boldsymbol{q}\right) \leq V\left(\boldsymbol{q}^{*}\right)-V(\boldsymbol{q}), \quad \forall \boldsymbol{q}^{*} .
$$

Furthermore, note that $V^{\prime}(\boldsymbol{q}, \delta \boldsymbol{q})=V(\boldsymbol{q}+\delta \boldsymbol{q})-V(\boldsymbol{q})$ for small $\boldsymbol{q}^{*}-\boldsymbol{q}=\delta \boldsymbol{q}$, because $V$ is polyhedral. With it, (3.6) becomes

$$
-\delta \boldsymbol{q}^{T}\left(\boldsymbol{M} \ddot{\boldsymbol{q}}^{+}-\boldsymbol{h}\right) \leq V^{\prime}(\boldsymbol{q}, \delta \boldsymbol{q})=V(\boldsymbol{q}+\delta \boldsymbol{q})-V(\boldsymbol{q}), \quad \forall \delta \boldsymbol{q} .
$$

\section{THE PRINCIPLE OF JOURDAIN}

The aim of this section is to write the subdifferential law (3.2) in terms of a velocity function $\Phi\left(\dot{\boldsymbol{q}}^{+}\right)$. By assumption, $V: \mathbb{R}^{f} \rightarrow \mathbb{R}$ is a polyhedral convex function of $\boldsymbol{q}$, i.e. a function whose epigraph is obtained by the intersection of a finite number of closed half spaces. An orthogonal projection to $\mathbb{R}^{f}$ of the intersections of the corresponding hyperplanes yields a division of $\mathbb{R}^{f}$ 
into a finite number of generalized polytopes. For any trajectory starting at $\boldsymbol{q}(t)$ in one of the polytopes, say $P_{0}$, we can find a sufficiently small $\tilde{t}>0$ such that the whole trajectory between $t$ and $t+\tilde{t}$ belongs to $P_{0}, \boldsymbol{q}\left(t^{*}\right) \in P_{0} ; t^{*} \in[t, t+\tilde{t})$. Note especially that $\boldsymbol{q}(t)$ may be at the boundary of $P_{0}$. Since discontinuities in the forces $\boldsymbol{f}(t)$ (see 3.2) and hence in the accelerations $\ddot{\boldsymbol{q}}(t)$ (see 3.1 ) arise only when passing transversally the boundary of a polytope, the trajectory between $t$ and $t+\tilde{t}$ is smooth and hence expandable into a Taylor series,

$$
\boldsymbol{q}(t+\tau)=\boldsymbol{q}(t)+\dot{\boldsymbol{q}}^{+}(t) \tau+\ldots, \quad 0 \leq \tau<\tilde{t}
$$

With it the subdifferential of $V$ at the point $\boldsymbol{q}(t+\tau)$ reads

$$
\partial V(\boldsymbol{q}(t+\tau))=\partial V\left(\boldsymbol{q}(t)+\dot{\boldsymbol{q}}^{+}(t) \tau\right)
$$

which is by the definition $(2.2)$

$$
\partial V\left(\boldsymbol{q}+\dot{\boldsymbol{q}}^{+} \tau\right)=\left\{-\overline{\boldsymbol{f}}^{+} \mid V^{\prime}\left(\boldsymbol{q}+\dot{\boldsymbol{q}}^{+} \tau, \boldsymbol{\xi}\right) \geq-\overline{\boldsymbol{f}}^{+T} \boldsymbol{\xi}, \quad \forall \boldsymbol{\xi}\right\}
$$

Since the directional derivative is positively homogeneous, and since $\left\|\boldsymbol{\xi} \tau^{2}\right\| \ll\left\|\dot{\boldsymbol{q}}^{+} \tau\right\|$ for small $\tau$, one can write

$$
\begin{aligned}
V^{\prime}\left(\boldsymbol{q}+\dot{\boldsymbol{q}}^{+} \tau, \boldsymbol{\xi}\right)= & \frac{1}{\tau^{2}} V^{\prime}\left(\boldsymbol{q}+\dot{\boldsymbol{q}}^{+} \tau, \boldsymbol{\xi} \tau^{2}\right) \\
= & \frac{1}{\tau^{2}}\left[V^{\prime}\left(\boldsymbol{q}, \dot{\boldsymbol{q}}^{+} \tau+\boldsymbol{\xi} \tau^{2}\right)-V^{\prime}\left(\boldsymbol{q}, \dot{\boldsymbol{q}}^{+} \tau\right)\right] \\
= & \frac{1}{\tau}\left[V^{\prime}\left(\boldsymbol{q}, \dot{\boldsymbol{q}}^{+}+\boldsymbol{\xi} \tau\right)-V^{\prime}\left(\boldsymbol{q}, \dot{\boldsymbol{q}}^{+}\right)\right] \\
\stackrel{\tau \downarrow 0}{\longrightarrow} & V^{\prime}\left(\boldsymbol{q}, \dot{\boldsymbol{q}}^{+}, \boldsymbol{\xi}\right)=\Phi^{\prime}\left(\dot{\boldsymbol{q}}^{+}, \boldsymbol{\xi}\right) \\
& \text { with } V^{\prime}\left(\boldsymbol{q}, \dot{\boldsymbol{q}}^{+}\right)=\Phi\left(\dot{\boldsymbol{q}}^{+}\right)
\end{aligned}
$$

where in the last line we have used the definition of the directional derivative (2.1), cp. [1]. With that result, (4.3) becomes

$$
\lim _{\tau \downarrow 0} \partial V(\boldsymbol{q}(t+\tau))=\left\{-\overline{\boldsymbol{f}}^{+} \mid \Phi^{\prime}\left(\dot{\boldsymbol{q}}^{+}, \boldsymbol{\xi}\right) \geq-\overline{\boldsymbol{f}}^{+T} \boldsymbol{\xi}, \quad \forall \boldsymbol{\xi}\right\}
$$

which is the definition of the subdifferential of $\Phi$, see (2.2):

$$
\lim _{\tau \downarrow 0} \partial V(\boldsymbol{q}(t+\tau))=\partial \Phi\left(\dot{\boldsymbol{q}}^{+}\right) .
$$

Summarizing, we see that the velocity potential $\Phi\left(\dot{\boldsymbol{q}}^{+}\right)$is the directional derivative of the potential function $V^{\prime}\left(\boldsymbol{q}, \dot{\boldsymbol{q}}^{+}\right)$considered as a function of $\dot{\boldsymbol{q}}^{+}$,

$$
\Phi\left(\dot{\boldsymbol{q}}^{+}\right)=V^{\prime}\left(\boldsymbol{q}, \dot{\boldsymbol{q}}^{+}\right)
$$

and by the result of Section 1, eq. (2.10), its subdifferential consists of the forces $\overline{\boldsymbol{f}}^{+}$for which

$$
-\overline{\boldsymbol{f}}^{+T} \dot{\boldsymbol{q}}^{+}=\sup \left(-\boldsymbol{f}^{+T} \dot{\boldsymbol{q}}^{+}\right), \quad-\overline{\boldsymbol{f}}^{+} \in \partial V(\boldsymbol{q}), \quad-\boldsymbol{f}^{+} \in \partial V(\boldsymbol{q}) .
$$

Note that $\Phi\left(\dot{\boldsymbol{q}}^{+}\right)$depends on $\boldsymbol{q}$ such that (cp. 4.4)

$$
V^{\prime}\left(\boldsymbol{q}+\varepsilon \dot{\boldsymbol{q}}^{+}, \dot{\boldsymbol{q}}^{+}, \boldsymbol{\xi}\right)=V^{\prime}\left(\boldsymbol{q}, \dot{\boldsymbol{q}}^{+}, \boldsymbol{\xi}\right)=\Phi^{\prime}\left(\dot{\boldsymbol{q}}^{+}, \boldsymbol{\xi}\right)
$$


for small $\varepsilon>0$ which is a property of polyhedral functions. Any other polyhedral convex function of $\dot{\boldsymbol{q}}^{+}$fulfilling (4.9), say $\tilde{V}\left(\boldsymbol{q}, \dot{\boldsymbol{q}}^{+}\right)$, can now be added to eq. (4.7) in order to take into account frictional or damping effects. The resulting $\Phi\left(\dot{\boldsymbol{q}}^{+}\right)$,

$$
\Phi\left(\dot{\boldsymbol{q}}^{+}\right)=V^{\prime}\left(\boldsymbol{q}, \dot{\boldsymbol{q}}^{+}\right)+\tilde{V}\left(\boldsymbol{q}, \dot{\boldsymbol{q}}^{+}\right)
$$

is then the velocity potential with which eq. (3.3) becomes

$$
-\left(\boldsymbol{M} \ddot{\boldsymbol{q}}^{+}-\boldsymbol{h}\right) \in \partial \Phi\left(\dot{\boldsymbol{q}}^{+}\right) .
$$

As in (3.4), $\partial \Phi\left(\dot{\boldsymbol{q}}^{+}\right)$can be stated as a variational inequality, cp. (4.5),

$$
-\overline{\boldsymbol{f}}^{+} \in \partial \Phi\left(\dot{\boldsymbol{q}}^{+}\right) \Leftrightarrow \Phi^{\prime}\left(\dot{\boldsymbol{q}}^{+}, \dot{\boldsymbol{q}}^{*+}-\dot{\boldsymbol{q}}^{+}\right) \geq-\overline{\boldsymbol{f}}^{+T}\left(\dot{\boldsymbol{q}}^{*+}-\dot{\boldsymbol{q}}^{+}\right), \quad \forall \dot{\boldsymbol{q}}^{*+}
$$

which leads together with (4.11) to the Principle of Jourdain,

$$
-\left(\dot{\boldsymbol{q}}^{*+}-\dot{\boldsymbol{q}}^{+}\right)^{T}\left(\boldsymbol{M} \ddot{\boldsymbol{q}}^{+}-\boldsymbol{h}\right) \leq \Phi^{\prime}\left(\dot{\boldsymbol{q}}^{+}, \dot{\boldsymbol{q}}^{*+}-\dot{\boldsymbol{q}}^{+}\right), \quad \forall \dot{\boldsymbol{q}}^{*+} .
$$

where the corresponding formulations (3.6) and (3.7) are also available since $\Phi\left(\dot{\boldsymbol{q}}^{+}\right)$is convex.

\section{THE PRINCIPLE OF GAUSS}

In this section the same steps as in Section 4 are performed in order to get the acceleration potential. The velocity potential $\Phi\left(\dot{\boldsymbol{q}}^{+}\right)$as a function of $\boldsymbol{q}$ and $\dot{\boldsymbol{q}}^{+}$, see (4.10), will be denoted by $\bar{V}$,

$$
\Phi\left(\dot{\boldsymbol{q}}^{+}\right)=\bar{V}\left(\boldsymbol{q}, \dot{\boldsymbol{q}}^{+}\right)
$$

where the property (4.9) is assumed to hold. With it the subdifferential of $\Phi$ at time $t+\tau$ can be written as

$$
\left.\partial \Phi\left(\dot{\boldsymbol{q}}^{+}\right)\right|_{t+\tau}=\partial_{\dot{q}^{+}} \bar{V}\left(\boldsymbol{q}(t+\tau), \dot{\boldsymbol{q}}^{+}(t+\tau)\right)
$$

which is by the definition $(2.2)$

$$
\partial_{\dot{q}^{+}} \bar{V}\left(\boldsymbol{q}(t+\tau), \dot{\boldsymbol{q}}^{+}(t+\tau)\right)=\left\{-\overline{\overline{\boldsymbol{f}}}^{+} \mid \bar{V}^{\prime}\left(\boldsymbol{q}(t+\tau), \dot{\boldsymbol{q}}^{+}(t+\tau), \boldsymbol{\xi}\right) \geq-\overline{\overline{\boldsymbol{f}}}^{+T} \boldsymbol{\xi}, \quad \forall \boldsymbol{\xi}\right\} .
$$

As in Section 4, the displacements $\boldsymbol{q}(t)$ and the velocities $\dot{\boldsymbol{q}}^{+}(t)$ can be expandet into a Taylor serie. Taking into account the additional property (4.9) the directional derivative in (5.3) becomes

$$
\bar{V}^{\prime}\left(\boldsymbol{q}(t+\tau), \dot{\boldsymbol{q}}^{+}(t+\tau), \boldsymbol{\xi}\right)=\Phi^{\prime}\left(\dot{\boldsymbol{q}}^{+}+\ddot{\boldsymbol{q}}^{+} \tau, \boldsymbol{\xi}\right)
$$

with which the subdifferential in (5.2) writes

$$
\left.\partial \Phi\left(\dot{\boldsymbol{q}}^{+}\right)\right|_{t+\tau}=\left\{-\overline{\overline{\boldsymbol{f}}}^{+} \mid \Phi^{\prime}\left(\dot{\boldsymbol{q}}^{+}+\ddot{\boldsymbol{q}}^{+} \tau, \boldsymbol{\xi}\right) \geq-\overline{\overline{\boldsymbol{f}}}^{+T} \boldsymbol{\xi}, \quad \forall \boldsymbol{\xi}\right\}
$$

This formulation, however, corresponds to eq. (4.3). Performing the same calculations as in eqs. (4.4)-(4.7) we are able to introduce the acceleration potential

$$
\Psi\left(\ddot{\boldsymbol{q}}^{+}\right)=\Phi^{\prime}\left(\dot{\boldsymbol{q}}^{+}, \ddot{\boldsymbol{q}}^{+}\right)
$$

from which we know that

$$
\left.\lim _{\tau \downarrow 0} \partial \Phi\left(\dot{\boldsymbol{q}}^{+}\right)\right|_{t+\tau}=\partial \Psi\left(\ddot{\boldsymbol{q}}^{+}\right)
$$


Substituting this expression into eq. (4.11) yields [2]

$$
-\left(\boldsymbol{M} \ddot{\boldsymbol{q}}^{+}-\boldsymbol{h}\right) \in \partial \Psi\left(\ddot{\boldsymbol{q}}^{+}\right)
$$

which is by (2.4) equivalent to the variational inequality

$$
-\left(\ddot{\boldsymbol{q}}^{*+}-\ddot{\boldsymbol{q}}^{+}\right)^{T}\left(\boldsymbol{M} \ddot{\boldsymbol{q}}^{+}-\boldsymbol{h}\right) \leq \Psi^{\prime}\left(\ddot{\boldsymbol{q}}^{+}, \ddot{\boldsymbol{q}}^{*+}-\ddot{\boldsymbol{q}}^{+}\right), \quad \forall \ddot{\boldsymbol{q}}^{*+} .
$$

This formulation is a generalization of the classical Principle of Gauss to systems with multivalued interaction laws resulting from polyhedral convex functions. Note especially that $\partial \Psi\left(\ddot{\boldsymbol{q}}^{+}\right) \subseteq \partial \Phi\left(\dot{\boldsymbol{q}}^{+}\right)$, and that the elements $-\overline{\boldsymbol{f}}^{+}$forming the first set are those from the second

set for which $-\overline{\bar{f}}^{+T} \ddot{\boldsymbol{q}}^{+}=\sup \left(\overline{\boldsymbol{f}}^{+T} \ddot{\boldsymbol{q}}^{+}\right)$. With eq. (5.8) and eq. (5.9) we have a direct access to the accelerations of the dynamical system which is the main advantage of that formulation. Examples of different interconnection laws as they appear in practical problems as well as many applications can be found in [7].

\section{THE PRINCIPLE OF LEAST CONSTRAINTS}

Equation (5.8) states the necessary and sufficient optimality conditions for a convex minimization problem. After integrating eq. (5.8) one arrives with

$$
f\left(\ddot{\boldsymbol{q}}^{+}\right)=\frac{1}{2} \ddot{\boldsymbol{q}}^{+T} \boldsymbol{M} \ddot{\boldsymbol{q}}^{+}-\boldsymbol{h}^{T} \ddot{\boldsymbol{q}}^{+}+\Psi\left(\ddot{\boldsymbol{q}}^{+}\right)
$$

which is indeed a strictly convex cost function, because $\boldsymbol{M}$ is symmetric and positive definite, and $\Psi\left(\ddot{\boldsymbol{q}}^{+}\right)$is convex. Moreover, since $f$ is strictly convex, the optimal solutions $\ddot{\boldsymbol{q}}^{+}$are unique. In classical mechanics this optimization problem is called the Principle of Least Constraints.

\section{ACKNOWLEDGEMENTS}

The author would like to thank Prof. P.D. Panagiotopoulos for his extremely helpful discussions, continuous encouragement and support. The financial support of the Alexander von Humboldt Foundation is gratefully acknowledged.

\section{REFERENCES}

1. ROCKAFELlAR R.T., Convex Analysis, Princeton University Press, Princeton, New Jersey, 1972.

2. GLOCKER CH., Dynamik von Starrkörpersystemen mit Reibung und Stössen, VDI-Fortschrittberichte Mechanik/Bruchmechanik, Reihe 18, Nr. 182, VDI-Verlag, Düsseldorf, 1995.

3. PANAGIOTOPOUlOS P.D., Hemivariational Inequalities, Springer Verlag Berlin, Heidelberg, 1993.

4. PANAGIOTOPOUlOS P.D., Ungleichungsprobleme und Differentialinklusionen in der Analyti-

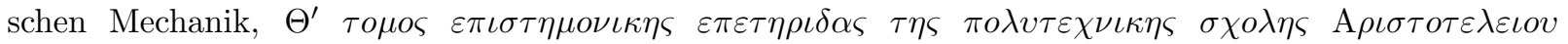
$\Pi \alpha \nu \varepsilon \pi \iota \sigma \tau \eta \mu \iota о v ~ \Theta \varepsilon \sigma \sigma \alpha \lambda o \nu \iota \kappa \eta \varsigma, \Theta \varepsilon \sigma \sigma \alpha \lambda o \nu \iota \kappa \eta, 1982$.

5. MOREAU J.J., Unilateral Contact and Dry Friction in Finite Freedom Dynamics, in Non-Smooth Mechanics and Applications, CISM Courses and Lectures, Vol. 302, Springer Verlag, Wien, 1988.

6. MOREAU J.J., Bounded Variation in Time, in Topics in Nonsmooth Mechanics (Edited by J.J. MOREAU, P.D. PANAGIOTOPOULOS and G. STRANG), Birkhäuser Verlag, Basel, 1988.

7. PFEIFFER F. \& GLOCKER CH., Multibody Dynamics with Unilateral Contacts, John Wiley \& Sons, New York, 1996. 